\title{
Is There a Role for Birch Pollen Immunotherapy on Concomitant Food Allergy? Riccardo Asero, MD
}

\author{
Address \\ Ambulatorio di Allergologia, Clinica San Carlo, Paderno Dugnano, MI, Italy \\ Email: r.asero@libero.it
}

Published online: 7 February 2015

(C) Springer International Publishing AG 2015

This article is part of the Topical Collection on Food Allergy

Keywords Birch pollen allergy · Oral allergy syndrome · Pollen-food allergy syndrome · Cross-reactivity · IgEmediated food allergy $\cdot$ Allergen specific immunotherapy

\section{Opinion statement}

Allergen-specific immunotherapy is the only treatment able to change the natural history of either respiratory or hymenoptera venom allergy. From an immunological point of view, there is no reason to believe that its clinical effects should be different in food allergies. However, due to the high prevalence of adverse events that accompanied the first attempts of injection immunotherapy with food extracts some 20 years ago, such treatment is presently non-available although a thoroughly standardized food extract for injection immunotherapy should theoretically not expose the patients to higher risks than airborne allergen extracts or venoms. Allergen-specific immunotherapy with birch pollen extract is an interesting model to investigate the effects of the treatment on plant-food allergies that occur as a consequence of cross-reactivity with the major allergen, Bet $v 1$. Although the interest for this field has partly settled during the last years, the available data suggest that an adequate dose of the birch pollen major allergen, Bet $v 1$, particularly if administered subcutaneously, is able reduce secondary plant food allergy also, although doses needed to exert such effect are probably higher than those required to reduce respiratory symptoms. The administration of higher doses of the major allergen without an increase of the risk of adverse reactions should be possible if modified hypoallergenic molecules obtained either by genetic engineering or chemical treatments are used.

\section{Introduction}

Allergen-specific immunotherapy (SIT) is presently the only treatment able to change the natural history of respiratory allergic disorders. Its efficacy is unquestionable and supported by a number of properly performed clinical studies $[1,2]$. Although many mechanisms are not fully elucidated, the clinical effect of SIT appears to rely mainly on the following:

(a) The induction of allergen-specific regulatory $\mathrm{T}$ and $\mathrm{B}$ cells that modulate the immune response shifting it towards a Th1 type (an effect possibly 
mediated by IL-12) and inducing immune tolerance by the production of IL-10, an immunosuppressive cytokine that leads to the suppression of allergen-specific effector T cells subsets, and

(b) The production of allergen-specific blocking IgG4 antibodies

The current knowledge about the mechanisms involved in allergen-specific immunotherapy has been recently reviewed [3]. Unfortunately, allergen-specific immunotherapy does not exist yet for the treatment of food allergy, and allergen avoidance remains the only way to prevent potentially life-threatening reactions in patients sensitized to foods. The lack of the development of SIT strategies for the cure of food allergies is probably largely due to the negative outcome of the first experi- mental attempts to desensitize peanut-allergic patients using aqueous extracts some 20 years ago in the USA. Such treatments proved clinically effective, in the sense that treated patients were able to tolerate increasing amounts of the offending food, but, probably due to the lack of proper allergen standardization, both the build-up phase and the maintenance protocol were associated with an unacceptably high rate of adverse systemic reactions that led to abandon this approach $[4,5]$. Studies on injection SIT using standardized chemically modified or genetically engineered food allergens are presently underway in different research centers, apparently with a low rate of adverse reactions, but, in the case they will be clinically successful, it will take years before these approaches become eventually available for the routine care of food-allergic patients.

\section{The pollen-food allergy syndrome}

The so-called pollen-food allergy syndrome is a particular type of IgE-mediated food allergy that occurs in patients with pollen allergy as a consequence of the cross-reactivity between pollen allergens and homologous proteins of plant-derived foods. The allergen proteins involved in this type of allergenic cross-reactivity are essentially three:

1) Bet v 1, the major birch pollen allergen belonging to pathogenesis-related proteins group 10 (PR-10). Proteins homologous, and hence potentially cross-reacting with Bet $\mathrm{v} 1$, are present in a large group of fruits and vegetables, including Rosaceae (apple, pear, peach, cherry, plum, apricot, almond, etc.), kiwi, tree nuts, peanut, and other legumes (soybean, bean, pea, chickpea, etc.), Apiaceae (celery, fennel, carrot, parsley), and several others.

2) Profilin, a largely cross-reacting 12 - to $15-\mathrm{kDa}$ protein present in all eucaryotic cells. It is a minor pollen allergen that sensitizes $10-20 \%$ of patients with pollen allergy. Profilin-hypersensitive patients may develop clinical allergy to a large array of fruits and vegetables, some of which (tomato, melon, watermelon, banana, and orange) are quite typical of this type of sensitization.

3) A hitherto not yet characterized mugwort pollen minor allergen responsible for the so called mugwort-celery-spice-syndrome, a pollen-food allergy mainly associated with celery, fennel, anise, and other spices.

The first two conditions are in most cases clinically characterized only by a local reaction called "oral allergy syndrome," since the food allergens involved in these IgE-mediated reactions are extremely labile and easily destroyed by heat and pepsin digestion, whereas the third condition is generally much more severe due to the stability of the sensitizing allergens.

\section{Pollen immunotherapy for food allergy}

The pollen-food allergy syndrome shows some advantages over primary food allergies that make it a unique opportunity to evaluate the effect of 
SIT on food allergies. First, the risk of adverse reactions is the same of the common SIT with airborne allergens as the treatment is carried out using well-characterized and standardized pollen extracts that are routinely used in thousands of allergic patients. In fact, the pollen-food allergy syndrome stems from a primary pollen sensitization [6], and food allergy is the consequence of the immunological cross-reactivity pollen and plant food allergens [7-10]; several lines of evidence suggest that virtually all allergenic epitopes of foods are present in pollen [7-9, 11]. The second, relevant advantage is that most food allergens involved in the pollen-food allergy syndrome are heat and pepsin sensitive. As a consequence, allergic symptoms induced by foods are in most cases mild and limited to oral allergy cavity (the so-called oral allergy syndrome, OAS), which allows oral food challenges to be performed without the fear of severe or even life-threatening allergic reactions [12].

In view of these features, there have been several studies which assessed the effects of pollen SIT on the pollen-food allergy syndrome during the past 25 years. Although also some studies assessing the effects of pollen SIT in patients hypersensitive to profilin have been carried out, in most cases, immunotherapy was based on birch pollen extracts as Bet $\mathrm{v} 1$, the major birch pollen allergen, is by far the most frequent cause of pollenfood cross-reactivity. Studies based on injection or sublingual immunotherapy can be distinguished.

\section{Injection immunotherapy with natural birch pollen extracts}

Moller carried out the study that pioneered this type of research in a pediatric cohort in 1989 [13]. Although the author disregarded the results as a failure due to the lack of a statistically significant difference between patients and controls, apple-induced OAS improved much in $43 \%(18 / 42)$ of patients submitted to injection SIT with birch pollen extract vs $14 \%(2 / 14)$ in the placebo group. Some 5 years later, a German group reported an improvement of apple-induced OAS in $45 \%$ $(9 / 20 \%)$ of those treated with birch pollen injection SIT [14]. This report was followed by an Italian study [15] that appeared towards the end of the twentieth century reporting that $84 \%$ (41/49) of patients treated with birch pollen subcutaneous immunotherapy experienced a marked reduction or a complete disappearance of the OAS following the ingestion of fresh apple; in contrast, no control (birch pollen allergic subjects with OAS not submitted to subcutaneous immunotherapy (SCIT)) noted any change in OAS. Interestingly, in most cases (43/49), a marked reduction or the total disappearance of skin reactivity to the fruit paralleled the clinical effect. A follow-up study carried out on the same cohort performed some years later found that the effect of birch pollen SCIT on apple-induced OAS was rather long lasting [16] and probably paralleled the effect of SCIT on respiratory allergy. A Polish study [17] confirmed these findings observing a positive effect of birch pollen SIT on apple allergy in $59 \%$ of cases. The year 2004 saw much interest on this issue. Bolhaar et al. [18] observed a reduction of apple allergy in $60 \%$ of their birch pollen-allergic patients after as short as 1 year of injection SIT and noted a decrease of skin reactivity to apple, 
hazelnut, and Mal d 1 in $>60 \%$ of patients. Bucher et al. [19] analyzed the effect of 1 year of injection SIT with birch pollen extract in subjects with OAS induced by apple or hazelnut. Patients showed an increased rate of skin reactivity to fresh apple, but $87 \%$ of them (vs only $8 \%$ of controls) could tolerate a significantly higher amount of offending food. Nonetheless, as the average amount of apple/hazelnut tolerated remained relatively small (32 g), the authors concluded that SIT has limited effects on OAS. Also in 2004, Hansen and co-workers obtained less brilliant results [20], as in their group of patients submitted to birch pollen SIT, the severity of food allergy did not change significantly despite a significant effect on seasonal rhinitis symptoms. There was some debate about the variability in the results of different studies that adopted similar treatment protocol and similar methods to measure the outcome. One of the suggested explanations was that the amount of allergen needed to produce an effect on secondary food allergy appeared to be higher than that required to reduce respiratory symptoms and that the concentration of the major allergen, Bet $\mathrm{v} 1$, in the extracts for injection SIT showed marked differences (even by a factor of 2) from one producer to another [21]. In effect, all studies performed in northern Europe with negative results employed for the SIT the commercially available birch pollen extract containing the lowest amount of Bet $\mathrm{v} 1$ among those whose concentration was known [21].

The variability of the results continued during the following years with a Polish study reporting a significant improvement of OAS in $50 \%$ of cases after SIT [22] and a study from the Netherlands reporting no effect on secondary hazelnut allergy [23]. In the same year (2011), another Italian study found that among patients submitted to injection immunotherapy with birch pollen, $25 \%$ developed complete tolerance to apple and further $37 \%$ showed an increase in the provocative dose [24]. The most recent contribution to this issue comes from Japan, where the oral allergy syndrome present in one patient responded dramatically well to a rush subcutaneous injection immunotherapy with birch pollen extract [25]. Altogether, though with some differences, results can still be regarded as encouraging.

\section{Sublingual immunotherapy with natural birch pollen extracts}

Sublingual allergen-specific immunotherapy (SLIT) has recently become a favorite way to perform immunotherapy for respiratory allergies in view of its good tolerability and convenience. Also in this case, there were studies that investigated to which extent birch pollen SLIT is effective on OAS induced by cross-reacting plant-derived foods. Unfortunately, the results have been much less brilliant than with injection immunotherapy.

Moller performed the first attempt in parallel with the injection immunotherapy study. Following SLIT with birch pollen extract, only $3 / 14$ (21\%) children reported a clinical effect on apple-induced OAS vs $2 / 14$ (14\%) with placebo [13]. After some years, Hansen and co-workers reported that in their group, SLIT did not change apple allergy similarly to injection immunotherapy [20]. Similarly, Kinaciyan et al. [26] did not 
notice any effect of birch pollen SLIT on apple allergy in spite of significant improvement of respiratory allergy in their nine patients. In contrast, more recently, Bergmann and co-workers [27] obtained much more favorable results, as they observed a reduction higher than $50 \%$ of apple allergy in 33/37 (89\%) patients after 12 months of SLIT.

\section{Immunotherapy with recombinant or genetically engineered birch pollen allergen}

With the advent of molecular biology techniques, studies based on engineered pollen allergens were carried out as well. Niederberger and coworkers were able to induce a reduction of OAS with a genetically modified birch pollen allergen containing hypoallergenic derivatives of Bet $\mathrm{v} 1$ (both Bet $v 1$ fragments and a hypoallergenic Bet $v 1$ trimer) in 7/25 treated patients [28]. In 2012, a couple of other studies appeared in the literature. In a preventive experimental study, a genetically engineered multi-allergen chimer obtained binding the whole Bet $\mathrm{v} 1$ molecule to immunodominant $\mathrm{T}$ cell epitopes of Api g 1 and Dau c 1 (the Bet $\mathrm{v} 1$ homologue allergen in celery and carrot, respectively) was intranasally applied to mice prior to sensitization with a mixture of Bet $\mathrm{v} 1$, Dau c 1 , and Api g 1. The pre-treatment was able to shift the immune response to Bet $\mathrm{v} 1$ and homologue food allergens towards a Th1 phenotype, thus preventing birch pollen-related food allergy [29•]. A multicenter trial aimed at investigating the effects of immunotherapy with a folded variant of recombinant Bet v 1 at a high dose $(80 \mu \mathrm{g}$; notably, the common commercial birch pollen extracts for injection immunotherapy contain 10$20 \mu \mathrm{g} / \mathrm{ml}$ of natural Bet $\mathrm{v} 1$ ) on birch-associated soy allergy has been announced, but the results have not been published yet [30].

\section{A critical analysis on available evidence}

Although the clinical immunotherapy trials reported above have produced a large amount of interesting results, it must be underlined that, with one exception [23], they were not randomized and placebo controlled. However, allergen immunotherapy is the only established curative treatment for respiratory allergy, and all the study patients underwent immunotherapy primarily to cure their respiratory allergies. Hence, one wonders whether administering placebo to severely allergic patients in order to measure a "favorable side effect" of SIT on secondary food allergy in the active arm of the study is ethically acceptable. Another point is that in most studies, efficacy on secondary food allergy was assessed by open oral challenges. Of course, since most patients suffered from oral allergy syndrome (i.e., subjective symptoms), a double-blind placebo-controlled food challenge (DBPCFC) would have been preferable. However, although they are considered the gold standard for the diagnosis of food allergy, DBPCFCs are extremely time consuming and demanding in terms of personnel, meal preparation, and facilities and pose a number of problems, particularly when the allergen proteins involved in allergic reactions are labile, as is the case with the pollen-food allergy syndrome [31]. By adopting both these strict criteria, we would be left with one single study that was randomized, double 
blind, and placebo controlled (DBPC) and assessed the effect on the linked hazelnut allergy by DBPCFC [23]; notably, this study found immunological changes but did not observe any clinical efficacy.

\section{Compliance with Ethics Guidelines}

\section{Conflict of Interest}

Riccardo Asero declares that he has no conflict of interest.

Human and Animal Rights and Informed Consent

This article does not contain any studies with human or animal subjects performed by any of the authors.

\section{References and Recommended Reading}

Papers of particular interest, published recently, have been highlighted as:

- Of importance

1. Bousquet J, Lockey RF, Malling HJ, editors. WHO position paper. Allergy immunotherapy: therapeutic vaccines for allergic diseases. Allergy 1998; 53(Suppl 44):1-42.

2. Durham SR, Walker SM, Varga EM, Jacobsen MR, O'Brien F, Noble W, et al. Long-term clinical efficacy of grass pollen immunotherapy. N Engl J Med. 1999;341:468-75.

3. Akdis M, Akdis CA. Mechanisms of allergen-specific immunotherapy: multiple suppressor factors at work in immune tolerance to allergens. J Allergy Clin Immunol. 2014;133:621-31.

4. Oppenheimer JJ, Nelson HS, Bock SA, Christiansen F, Leung DYM. Treatment of peanut allergy with rush immunotherapy. J Allergy Clin Immunol. 1992;90:256-62.

5. Nelson HS, Lahr J, Rule R, Bock SA, Leung DYM. Treatment of anaphylactic sensitivity to peanuts by immunotherapy with injections of aqueous peanut extract. J Allergy Clin Immunol. 1997;99:744-51.

6. Valenta R, Kraft D. Type I allergic reactions to plantderived foods: a consequence of primary sensitization to pollen allergens. J Allergy Clin Immunol. 1996;97:893-8.

7. Ebner C, Birkner T, Valenta R, Rumpold H, Breitenbach $\mathrm{M}$, Scheiner O, et al. Common epitopes of birch pollen and apple. Studies by Western and Northern blot. J Allergy Clin Immunol. 1991;88:588-94.

8. Vieths S, Janek K, Aulepp H, Petersen A. Isolation and characterization of the $18 \mathrm{kDa}$ major apple allergen and comparison with the major birch pollen allergen (Bet v 1). Allergy. 1995;50:421-30.

9. Vanek-Krebiz M, Hoffmann-Sommergruber K, Laimer da Camara Machado M, Ebner C, Kraft D, Scheiner O, et al. Cloning and sequencing of Mal d 1, the major allergen from apple (Malus domestica), and its immunological relationship to Bet $\mathrm{v} 1$ the major birch pollen allergen. Biochem Biophys Res Commun. 1995;214:538-51.

10. Valenta R, Duchene M, Ebner C, Valent P, Sillaber C, Deviller $\mathrm{P}$, et al. Profilins constitute a novel family of plant pan-allergens. J Exp Med. 1992;175:377-85.

11. Halmepuro L, Vuontela K, Kalimo K, Bjorksten F. Cross-reactivity of IgE antibodies with allergens in birch pollen, fruits and vegetables. Int Arch Allergy Appl Immunol. 1984;74:235-40.

12. Anhoej C, Backer V, Nolte H. Diagnostic evaluation of grass- and birch-allergic patients with oral allergy syndrome. Allergy. 2001;56:548-52.

13. Moller C. Effect of pollen immunotherapy on food hypersensitivity in children with birch pollinosis. Ann Allergy. 1989;62:343-5.

14. Herrmann D, Henzgen M, Frank E, Rudeschko O, Jager L. Effect of hyposensitization for tree pollinosis on associated apple allergy. J Investig Allergol Clin Immunol. 1995;5:259-67.

15. Asero R. Effect of birch pollen-specific immunotherapy on apple allergy in birch pollen-hypersensitive patients. Clin Exp Allergy. 1998;28:1368-73.

16. Asero R. How long does the effect of birch pollen injection SIT on apple allergy last? Allergy. 2003;58:435-8.

17. Modrzynski M, Zawisza E, Rapiejko P, Przybylski G. Specific-pollen immunotherapy in the treatment of oral allergy syndrome in patients with tree pollen hypersensitivity. Przegl Lek. 2002;59:1007-10.

18. Bolhaar ST, Tiemessen MM, Zuidmeer L, van Leeuwen A, Hoffmann-Sommergruber K, Bruijnzeel-Koomen CA, et al. Efficacy of birch-pollen immunotherapy on cross-reactive food allergy confirmed by skin tests and double-blind food challenges. Clin Exp Allergy. 2004;34:761-9. 
19. Bucher X, Pichler WJ, Dahinden CA, Helbling A. Effect of tree pollen specific, subcutaneous immunotherapy on the oral allergy syndrome to apple and hazelnut. Allergy. 2004;52:1272-6.

20. Hansen KS, Khinchi MS, Skov PS, Bindslev-Jensen C, Poulsen L, Malling HJ. Food allergy to apple and specific immunotherapy with birch pollen. Mol Nutr Food Res. 2004;48:441-8.

21. Asero R. Effects of birch pollen SIT on apple allergy: a matter of dosage? Allergy. 2004;59:1269-71.

22. Czarnecka-Operacz M, Jenerowicz D, Silny W. Oral allergy syndrome in patients with airborne pollen allergy treated with specific immunotherapy. Acta Dermatovenerol Croat. 2008;16:19-24.

23. Van Hoffen E, Peeters HA, van Neerven RJ, Zuidmeer L, et al. Effect of birch pollen-specific immunotherapy on birch pollen-related hazelnut allergy. J Allergy Clin Immunol. 2011;127:100-1.

24. Mauro M, Russello M, Incorvaia C, Gazzola G, Frati F, Moingeon $\mathrm{P}$, et al. Birch-apple syndrome treated with birch pollen immunotherapy. Int Arch Allergy Immunol. 2011;156:416-22.

25. Okamoto Y, Kurihara K. A case of oral allergy syndrome whose symptoms were dramatically improved after rush subcutaneous injection immunotherapy with pollen extracts of birch. Arerugi. 2012;61:652-8.

26. Kinaciyan T, Jahn-Schmid B, Radakovics A, Zwölfer B, Schreiber C, Francis JN, et al. Successful sublingual immunotherapy with birch pollen has limited effects on concomitant food allergy to apple and the immune response to the Bet v 1 homolog Mal d 1. J Allergy Clin Immunol. 2007;119:937-43.

27. Bergmann KC, Wolf H, Schnitker J. Effect of pollenspecific sublingual immunotherapy on oral allergy syndrome: an observational study. World Allergy Organ J. 2008;1:79-84.

28. Niederberger V, Reisinger J, Valent P, Krauth MT, Pauli $\mathrm{G}$, et al. Vaccination with genetically modified birch pollen allergens: immune and clinical effects on oral allergy syndrome. J Allergy Clin Immunol. 2007;119:1013-6.

29. Hoflehner E, Hufnagl K, Schabussova I, Jasinska J, Hoffmann-Sommergruber K, Bohle B, et al. Prevention of birch pollen related food allergy by mucosal treatment with multi allergen-chimers in mice. PLoS One. 2012;7:e39409.

This is the first "preventive" study carried out so far, and might represent a model for future studies in humans. Another interesting aspect is that an engineered allergen molecule was used, which underlines how immunogenic/non allergenic molecules may work in this sense.

30. Treudler R, Simon JC. Severe soy allergy in adults. Is there a role for specific immunotherapy? Hautarzt. 2012;63:307-12.

31. Asero R, Fernandez-Rivas M, Knulst AC, BruijnzeelKoomen CA. Double-blind, placebo-controlled food challenge in adults in everyday clinical practice: a reappraisal of their limitations and real indications. Curr Opin Allergy Clin Immunol. 2009;9:379-85. 